\title{
Low-to-High Cross-Frequency Coupling in the Electrical Rhythms as Biomarker for Hyperexcitable Neuro-Glial Networks of the Brain
}

\author{
Vasily Grigorovsky, Berj L. Bardakjian \\ Version Post-print/accepted manuscript \\ Citation Grigorovsky, Vasily \& Bardakjian, Berj. (2017). Low-to-High Cross- \\ (published version) Frequency Coupling in the Electrical Rhythms as Biomarker for \\ Hyperexcitable Neuro-Glial Networks of the Brain. IEEE Transactions \\ on Biomedical Engineering. PP. 1-1. 10.1109/TBME.2017.2757878. \\ Publisher's Statement C 2017 IEEE. Personal use of this material is permitted. \\ Permission from IEEE must be obtained for all other uses, in any \\ current or future media, including reprinting/republishing this \\ material for advertising or promotional purposes, creating new \\ collective works, for resale or redistribution to servers or lists, or \\ reuse of any copyrighted component of this work in other works.
}

How to cite TSpace items

Always cite the published version, so the author(s) will receive recognition through services that track citation counts, e.g. Scopus. If you need to cite the page number of the author manuscript from TSpace because you cannot access the published version, then cite the TSpace version in addition to the published version using the permanent URI (handle) found on the record page.

This article was made openly accessible by $U$ of 'T Faculty.

Please tell us how this access benefits you. Your story matters. 


\title{
Low-to-High Cross-Frequency Coupling in the Electrical Rhythms as Biomarker for Hyperexcitable Neuro-Glial Networks of the Brain
}

\author{
Vasily Grigorovsky, Member, IEEE, Berj L. Bardakjian, Member, IEEE
}

\begin{abstract}
Objective: One of the features used in the study of hyperexcitablility are high frequency oscillations (HFOs, $>\mathbf{8 0 H z}$ ). HFOs have been reported in the electrical rhythms of the brain's neuro-glial networks under physiological and pathological conditions. Cross-frequency coupling (CFC) of HFOs with low frequency rhythms was used to identify pathologic HFOs (pHFOs) (i) in the epileptogenic zones of epileptic patients, and (ii) as a biomarker for the severity of seizure-like events in genetically modified rodent models. We describe a model to replicate reported CFC features extracted from recorded local field potentials (LFPs) representing network properties. Methods: This study deals with a 4-unit neuro-glial cellular network model where each unit incorporates pyramidal cells, interneurons and astrocytes. Three different pathways of hyperexcitability generation - $\mathrm{Na}^{+}-\mathrm{K}^{+}$ATPase pump, glial potassium clearance, and potassium afterhyperpolarization channel - were used to generate LFPs. Changes in excitability, average spontaneous electrical discharge (SED) duration and CFC were then measured and analyzed. Results: Each parameter caused an increase in network excitability and the consequent lengthening of the SED duration. Short SEDs showed CFC between HFOs and theta oscillations (4-8 $\mathrm{Hz})$, but in longer SEDs the low frequency changed to the delta range (1-4 Hz). Conclusion: Longer duration SEDs exhibit CFC features similar to those reported by our team. Significance: (i) Identifying the exponential relationship between network excitability and SED durations, (ii) highlighting the importance of glia in hyperexcitability (as they relate to extracellular potassium), and (iii) elucidation of the biophysical basis for CFC coupling features.
\end{abstract}

Index Terms-Epilepsy, Neuro-glial network, Hyperexcitability, Cellular modelling, Cross-frequency coupling, High frequency oscillations, $\mathrm{Na}^{+}-\mathrm{K}^{+}$ATPase pump, Glial potassium clearance, Potassium afterhyperpolarization

\section{INTRODUCTION}

$\mathbf{H}$ YPEREXCITABILITY describes the state of highly active synchronized neuronal networks and it has been implicated in many neurological disorders [1]. In epilepsy, hyperexcitability is frequently characterized by recurrent spontaneous electric discharges (SED) - rapid bursts of membrane depolarization which can be generally classified as long (greater than two seconds) or short in duration [2]. In rodent models of Rett's syndrome neuronal network states

Research is generously supported by Canadian Institutes of Health Research (CIHR) and Natural Sciences and Engineering Research Council (NSERC)

V. Grigorovsky is with the Institute of Biomaterials and Biomedical Engineering, University of Toronto, ON M5S3G9 Canada (e-mail: vasily.grigorovsky@utoronto.ca).

B. L. Bardakjian is with the Institute of Biomaterials and Biomedical Engineering, University of Toronto, ON M5S3G9 Canada (e-mail: berj.bardakjian@utoronto.ca). dominated by long duration SEDs were indicative of more severe outcomes and the improvement in the outcomes was associated with shortening of the average SED duration [2]. Thus, understanding mechanisms that control the duration of SED could help to determine their causal effect on seizure severity, and used as biomarkers for epileptiform activity.

One biomarker for the duration and the level of severity of SEDs in rodent models is cross-frequency coupling (CFC) between low and high frequency oscillations (LFOs and HFOs respectively) [2]. Furthermore, our team has found that certain CFC features can be used to identify epileptogenic zones in patients, specifically the presence of the strong coupling of HFOs $(>80 \mathrm{~Hz})$ with the delta rhythm $(1-4 \mathrm{~Hz})$ in adults [3], which was not seen during non-seizure states or outside epileptogenic zones. Although HFOs are always present in an active brain, they differ based on whether they are physiological or pathological (pHFO) in origin. pHFO mechanisms and properties are of particular interest, since they can shed light on the nature of epileptic seizures, and they can be identified based on their CFC - for example in contrast to pHFOs found by our team, physiological high frequency oscillations are frequently modulated by theta $(4-8 \mathrm{~Hz})$ [4]. Therefore, it is reasonable to conclude that CFC features can be used to identify and evaluate epileptogenic activity in neuronal networks; however, the relationship between dominant frequencies and frequency couplings, and the duration of SED is not conclusively known.

Computational models used to understand mechanisms behind epileptogenesis allow for more precise control of the environment and provide an opportunity to sidestep the ethical and methodological problems associated with in vivo experimental studies. However, most of them focus on either individual neuron studies with complex morphology or network effects with simplistic neuronal representations, and largely do not take into account the potential contribution from glial cells which account for more than half of all brain cells. Astrocytes, the most prominent glial subgroup, were originally thought to provide support and protection to the surrounding neurons due to their role in glucose metabolism and extracellular ion concentration maintenance. However recent experimental evidence revealed that astrocytes play a far greater role in neuronal circuits [5]. They have been shown to be closely involved in synapse formation and management [6], which can be seen as crucial for controlling the level of excitability of the network. Additionally, they moderate and alter neuronal firing patterns in response to local environment conditions [7]. Furthermore, astrocytic dysfunction is correlated with neuro- 


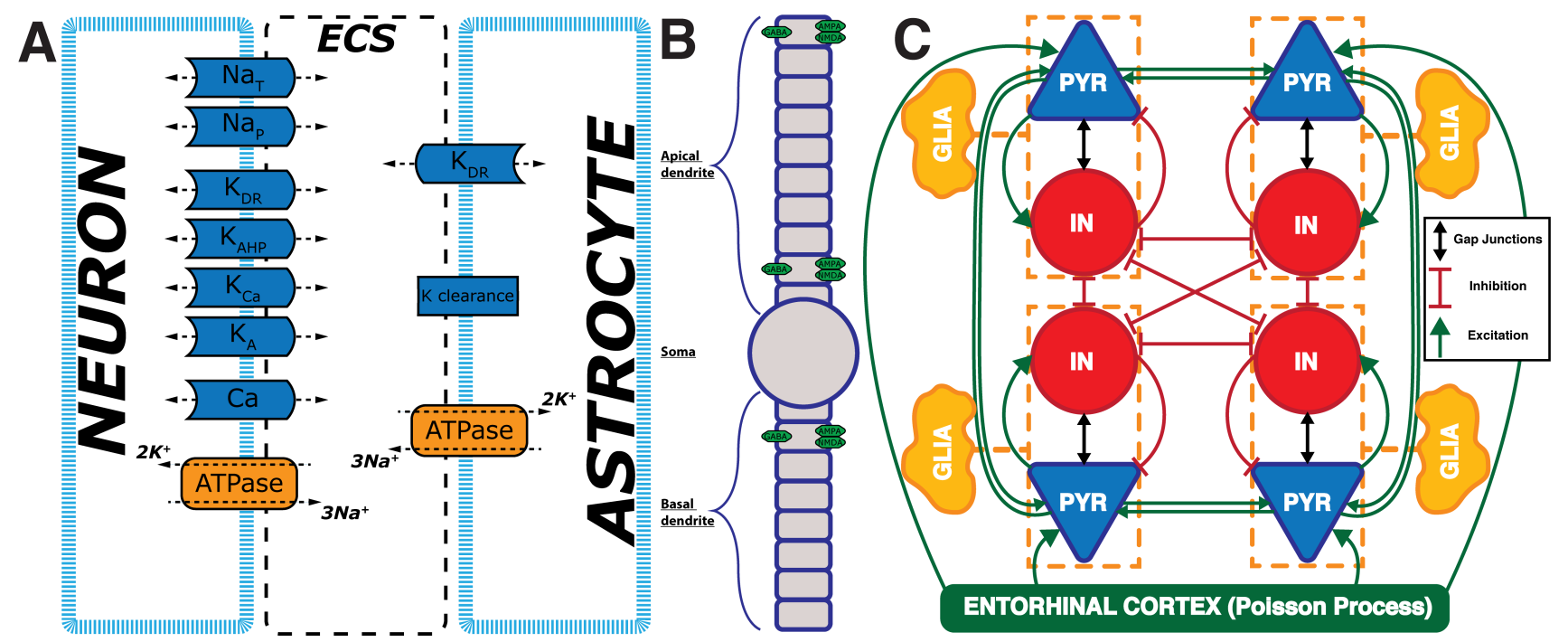

Fig. 1. A) Generic neuron compartment and astrocyte with appropriate channels and pumps. B) Single neuron with compartment-specific synaptic channels. C) The modelled network, with excitation and inhibition pathways, glial clearance domains, and the influence of entorhinal cortex.

physiological disorders such as epilepsy [5].

Several physiological changes consistently appear in hyperexcitable networks, such as decreased expression of potassium afterhyperpolarisation [8]. In addition, experimental studies have explored the notion that elevated extracellular potassium concentration can generate seizure-like activity [9] since it can significantly increase potassium channel reversal potential, making these channels depolarizing. Normally, potassium released into the extracellular space is reabsorbed via $\mathrm{Na}^{+}-\mathrm{K}^{+}$ ATPase pump [5]. However, during periods of higher neural activity, sodium-potassium pumps get overwhelmed and extracellular potassium concentration is maintained through several other mechanisms. One such mechanism is spatial diffusion of extracellular potassium to areas of lower concentration [10]. Another mechanism is via glial potassium moderation, either using inwardly rectifying Kir4.1 channels [10] or $\mathrm{Na}^{+}-\mathrm{K}^{+}-\mathrm{Cl}^{-}$ cotransporter NKCC1 [11].

Since the early 2000's, several groups started shifting their modelling research focus from neuronal to neuro-glial networks to investigate effects that astrocytes can play in network dynamics [12], [13]. Some groups looked into how glial $\mathrm{Na}^{+}-$ $\mathrm{K}^{+}$ATPase pump affected neuronal hyperexcitability [11]; others investigated the interaction between neuronal pumps and glial potassium buffering on maintaining extracellular potassium concentrations [9], [14]-[16]. More in-depth analysis of astrocytic interactions has been briefly investigated by adding inwardly rectifying potassium Kir4.1 channels [7] and $\mathrm{Na}^{+}-\mathrm{K}^{+}-\mathrm{Cl}^{-}$cotransporter NKCC1 [11]. Moreover, astrocytes also have been shown to play a crucial role in maintaining the extracellular and - indirectly - cell volumes, both due to potassium interactions and Aquaporin 4 channels [17]. Finally, astrocytes have also been included in the models of oxygen effects on seizure dynamics [18]. Still, despite this research, the influence of these mechanisms on the seizure severity and frequency dynamics has not been conclusively established.

In this study we propose that the state of hyperexcitability achieved by manipulating some of the pathways described above and characterized by long duration SEDs shows a significant increase in delta-HFO coupling, which is consistent with both the biomarkers for epileptogenic zones and seizure severity associated with long duration SEDs. We achieve that by creating the neuro-glial model and validating it by comparing its output with local field potential (LFP) recordings from in vitro studies that our lab has done. Previous work that our team has done has shown that transmembrane potential and LFP recordings demonstrate similar frequencies [19], so we feel justified in utilizing cellular neuro-glial model to investigate CFC dynamics.

\section{Methods}

In order to evaluate this hypothesis, we have developed a network model of a typical neuronal circuit found in the hippocampal CA3 region, consisting of four interconnected three-cell neural units (see Fig. 1C). Each unit consisted of a pyramidal cell, a fast-spiking interneuron, and an associated astrocyte. Four units were chosen, as that was previously found to be a minimal number of active neurons to be able to generate network effects [20]. As has been done in other studies, the rest of the network was represented by stochastic Poisson process [21] interacting with all of the pyramidal cells with a rate of $10 \mathrm{~Hz}$ [22]. Individual circuits also interacted with each other through inhibitory (interneurons) and excitatory (pyramidal cells) connections. Gap junctions between interneurons and axo-axonal junctions in pyramidal cells were also included. In order to avoid network entrainment, connections within the model were varied in strength, with $15 \%$ of connection strength in standard deviation [24]. Additionally, since pyramidal cell axons are myelinated, whereas interneuron axons are not, varying delay in the excitatory/inhibitory connections was introduced to mimic the speed of signal propagation - a feature of the GENESIS simulation platform [25]. 
TABLE I

SUMMARY OF THE CURRENT EQUATIONS AND THE PARAMETERS FOR GLIAL POTASSIUM BUFFERING. ALL OF THE EQUATIONS ARE ADAPTED FROM [14] UNLESS STATED OTHERWISE

\begin{tabular}{|c|c|c|}
\hline Term & Expression & Description \\
\hline $\boldsymbol{I}_{\boldsymbol{K}, \boldsymbol{d} \boldsymbol{r}}$ & $g_{K, d r} m_{K, d r}^{2}\left(V-E_{K}\right)$ & Delayed rectifier potassium current \\
\hline $\boldsymbol{I}_{\boldsymbol{K}, \boldsymbol{a}}$ & $g_{K, a} m_{K, a}^{2} h_{K, a}\left(V-E_{K}\right)$ & Regular (A-type) potassium current \\
\hline $\boldsymbol{I}_{\boldsymbol{K}, \boldsymbol{c}}$ & $g_{K, c} m_{K, c}\left(V-E_{K}\right) * \min \left(1,[C a]_{i} / 250\right)$ & Calcium-dependent potassium current \\
\hline $\boldsymbol{I}_{\boldsymbol{K}, \boldsymbol{a h} \boldsymbol{p}}$ & $g_{K, a h p} m_{K, a h p}\left(V-E_{K}\right)$ & Afterhyperpolarization potassium current \\
\hline $\boldsymbol{I}_{\boldsymbol{N a}}$ & $g_{N a} m_{N a}^{3} h_{N a}\left(V-E_{N a}\right)$ & Transient sodium current \\
\hline $\boldsymbol{I}_{\boldsymbol{N a}, \boldsymbol{p}}$ & $g_{N a, p} m_{N a, p}^{2} h_{N a, p}\left(V-E_{N a}\right)$ & Persistent sodium current \\
\hline $\boldsymbol{I}_{\boldsymbol{C a}}$ & $g_{C a} m_{C a}^{2}\left(V-E_{C a}\right)$ & Calcium current [23] \\
\hline $\boldsymbol{I}_{\boldsymbol{l e a k}}$ & $g_{l e a k}\left(V-E_{l e a k}\right)$ & Leak current \\
\hline $\boldsymbol{I}_{\boldsymbol{p u m p}}$ & $I_{m a x}\left(1 /\left(1+K_{\alpha} /[K]_{o}\right)\right)^{2}\left(1 /\left(1+N a_{\alpha} /[N a]_{i}\right)\right)^{3}$ & Sodium-potassium ATPase pump current [12] \\
\hline $\boldsymbol{k}_{\boldsymbol{O N}}$ & $k_{O F F} /\left(1+\exp \left(\left([K]_{T H}-[K]_{o}\right) / 1.15\right)\right)$ & Glial potassium buffering binding rate \\
\hline $\boldsymbol{k}_{\boldsymbol{O F F}}$ & 0.008 & Glial potassium buffering unbinding rate \\
\hline
\end{tabular}

\section{A. Pyramidal Cell and Interneuron Models}

Each neural unit consisted of two neurons - a pyramidal cell and an interneuron. Both neurons consisted of 19 compartments as per Traub's specification for an unbranched compartmentalized model [26] - 10 for the apical dendrite, 8 for basal dendrite, and a soma (see Fig. 1B). For each compartment, the evolution of membrane voltage was calculated using the following equation [11], [14]:

$$
C_{m} \frac{d V_{m}}{d t}=-I_{\text {int }}-I_{\text {leak }}-I_{\text {pump }}-X
$$

where $V_{m}$ is the membrane voltage, $I_{i n t}$ is the sum of intrinsic currents, $X$ term accounts for cross-compartment coupling (modelled as $g_{\text {compartment }} \Delta V$ ), and $I_{\text {pump }}$ is the current due to the sodium-potassium pump. All ionic currents follow the same dynamics with some minor variations:

$$
\begin{gathered}
I_{a}=g_{a} m_{a}^{b} h_{a}^{c}\left(V-E_{a}\right) \\
d x / d t=\alpha_{x}(1-x)-\beta_{x} x
\end{gathered}
$$

where $g$ is the maximal conductance, $m$ is the activating variable, $h$ is the inactivating variable, and $E$ is the equilibrium potential. Equation 3 shows general pattern for activation and inactivation kinetics. The precise equations used for current dynamics modelling can be seen in table 1 , the rate equations in table 2, and the parameters and initial values used are listed in table 3 . The initial values of all activating/inactivating variables were determined using the following equation:

$$
m_{x, \infty}=\alpha_{x} /\left(\alpha_{x}-\beta_{x}\right)
$$

Furthermore, unlike many other modelling studies, equilibrium potentials for various ions were dynamic, and calculated using the standard Nernst equation (where $z$ is the valence of the ion):

$$
E=26.64 / z * \ln \left([\text { Ion }]_{o} /[\text { Ion }]_{i}\right)
$$

Channels were modelled with Gaussian distribution, with maximal channel density (and as such - conductance) at the soma and going down to zero at the terminal dendrites, since this was found to be adequate in comparative modelling studies [27]. The only exception to this is the distribution of synaptic receptors - AMPA, NMDA, and GABA. Synaptic receptors were added to allow for network interactions in addition to neuronal responses to and effects on extracellular ion concentrations. They produced synaptic current $I_{s y n}$ which was treated as the stimulation current, in addition to intrinsic dynamics of neurons. These receptors were located on the terminal dendritic compartments, as well as the somatic compartment to allow for perisomatic inhibition. All of the synaptic receptors were modelled as double exponentials:

$$
\begin{gathered}
I_{\text {syn }}=g_{\text {syn }} \gamma\left(V-E_{\text {syn }}\right) \\
\gamma=\frac{a\left[\exp \left(-t / \tau_{1}\right)-\exp \left(-t / \tau_{2}\right)\right.}{b} \\
b_{A M P A}=b_{G A B A}=1 \\
b_{N M D A}=1+\frac{[M g]_{o} * \exp (-0.062 V)}{3.57}
\end{gathered}
$$

where $a$ is the normalization factor to ensure that $\gamma$ is between 0 and 1 . The factor $b_{x}$ is used to differentiate between more straightforward AMPA/GABA receptors, and NMDA receptor which has a voltage-dependent magnesium block that plays an important role in synaptic transmission.

\section{B. Astrocyte Model and Ion Balance}

In the model every astrocyte was represented as a single compartment. Overall the compartment followed similar voltage dynamics equations as neuronal compartments, however it had fewer currents present - delayed rectifier potassium channel $\left(\mathrm{K}_{\mathrm{DR}}\right)$ [7], $\mathrm{Na}^{+}-\mathrm{K}^{+}$ATPase pump, and leak currents [11]. $K_{D R}$ channel dynamics were similar to the neuronal one, however its effective voltage was hyperpolarized by $50 \mathrm{mV}$ with respect to the membrane voltage, as per [7]. The main purpose of the astrocyte in this model, however, was to help 
TABLE II

SUMMARY OF THE RATE EQUATIONS, ADAPTED FROM [14] EXCEPT WHERE NOTED

\begin{tabular}{|c|c|c|}
\hline Term & Expression & Description \\
\hline$\alpha_{m_{K, d r}}$ & $0.016(-V-34.9) /(\exp (-0.2 V-6.98)-1)$ & Forward rate for $m_{K, d r}$ \\
\hline$\beta_{m_{K, d r}}$ & $0.25 \exp (-0.025 V-1.25)$ & Backward rate for $m_{K, d r}$ \\
\hline$\alpha_{m_{K, a}}$ & $0.02(-V-56.9) /(\exp (-0.1 V-5.69)-1)$ & Forward rate for $m_{K, a}$ \\
\hline$\beta_{m_{K, a}}$ & $0.0175(V+29.9) /(\exp (0.1 V+2.99)-1)$ & Backward rate for $m_{K, a}$ \\
\hline$\alpha_{h_{K, a}}$ & $0.016 \exp (-0.056 V-4.61)$ & Forward rate for $h_{K, a}$ \\
\hline$\beta_{h_{K, a}}$ & $0.5 /(\exp (-0.2 V-11.98)+1)$ & Backward rate for $m_{K, a}$ \\
\hline$\alpha_{m_{K, c}}$ & $\exp ((V-10) / 11-(V-6.5) / 27) / 18.975$ & Forward rate for $m_{K, c}$ \\
\hline$\beta_{m_{K, c}}$ & $2 \exp ((-V+6.5) / 27)$ & Backward rate for $m_{K, c}$ \\
\hline$\alpha_{m_{K, a h p}}$ & $\min \left(0.2 * 10^{-4} *[\mathrm{Ca}]_{i}, 0.01\right)$ & Forward rate for $m_{K, a h p}$ \\
\hline$\beta_{m_{K, a h p}}$ & 0.001 & Backward rate for $m_{K, a h p}$ \\
\hline$\alpha_{m_{N a}}$ & $0.32(-V-51.9) /(\exp (-0.25 V-12.975)-1)$ & Forward rate for $m_{N a}$ \\
\hline$\beta_{m_{N a}}$ & $0.28(V+24.89) /(\exp (0.2 V+4.978)-1)$ & Backward rate for $m_{N a}$ \\
\hline$\alpha_{h_{N a}}$ & $0.128 \exp (-0.056 V-2.94)$ & Forward rate for $h_{N a}$ \\
\hline$\beta_{h_{N a}}$ & $4 /(\exp (-0.2 V-6)+1)$ & Backward rate for $m_{N a}$ \\
\hline$\alpha_{m_{N a, p}}$ & $1 / 6(\exp (-0.143 V-5.67)+1)$ & Forward rate for $m_{N a, p}$ \\
\hline$\beta_{m_{N a, p}}$ & $\exp (-0.143 V-5.67) / 6(\exp (-0.143 V-5.67)+1)$ & Backward rate for $m_{N a, p}$ \\
\hline$\alpha_{h_{N a, p}}$ & $5.12 * 10^{-8} \exp (-0.056 V-2.94)$ & Forward rate for $h_{N a, p}$ \\
\hline$\beta_{h_{N a, p}}$ & $1.6 * 10^{-6} /(\exp (-0.2 V-8)+1)$ & Backward rate for $m_{N a, p}$ \\
\hline$\alpha_{m_{C a}}$ & $1.6 /(\exp (-0.072 V+4.68)+1)$ & Forward rate for $m_{C a}[23]$ \\
\hline$\beta_{m_{C a}}$ & $\exp (0.02 V-1.022) / 6(\exp (0.2 V-10.22)-1)$ & Backward rate for $m_{C a}$ [23] \\
\hline
\end{tabular}

maintain extracellular potassium concentration. Although the electroneutrality of the astrocytic membrane was maintained with the balance of $\mathrm{K}_{\mathrm{DR}}$ and $\mathrm{Na}^{+}-\mathrm{K}^{+}$ATPase pump, the main acting mechanism was the potassium buffering due to numerous glial effects. In general, the concentration of each ion in the model was determined by leak and intrinsic currents and, in case of potassium - glial buffering. Overall the concentrations were governed by the following equations:

$$
\begin{gathered}
d[K]_{i} / d t=-D\left(\sum I_{K}-2 I_{\text {pump }}\right) \\
d[K]_{o} / d t=-2\left(d[K]_{i} / d t\right)-G- \\
D\left(I_{K_{d r}}+2 I_{\text {pump }}\right) \\
G=\frac{-d[B]}{d t}=k_{O N}[K]_{o}[B]-k_{O F F}\left([B]_{T}-[B]\right) \\
d[N a]_{i} / d t=D\left(\sum I_{N a}-3 I_{\text {pump }}\right) \\
d[N a]_{o} / d t=-2\left(d[N a]_{i} / d t\right) \\
d[C a]_{i} / d t=D * I_{C a}-[C a]_{i} / \tau
\end{gathered}
$$

where $D$ is a conversion factor from the particular current to concentration, and $G$ is glial potassium buffering (equation (12)). The $D$ factor was obtained as $D=\frac{k}{F d}$, where $k$ is the conversion factor for change in concentration, $d$ is rough ratio of surface area to volume, and $F$ is Faraday's constant, and the factor was approximated to be $6.9^{*} 10^{-4} \mathrm{~mol} / \mathrm{s}^{*} \mathrm{~A}$ [14]. Factor of 2 is present in front of both $d[K]_{i} / d t$ and $d[N a]_{i} / d t$ as there are two neurons in each unit - pyramidal cell and interneuron. In order to simplify the model, calcium ion dynamics (equation (15)) were simplified to only track the intracellular ionic concentration. Several additional assumptions have been made about the network and ion concentration change to simplify the model. It was assumed that the overall cation current is zero, meaning that $\mathrm{Na}^{+}$and $\mathrm{K}^{+}$currents are equal in magnitude. Another assumption was that there is a charge neutrality of extracellular space. Finally, glial ion concentrations were assumed to be constant (since glial cells form syncytium [28]).

\section{Data Collection and Analysis}

In order to test the hypothesis, the model was used to generate two kinds of data - transmembrane potentials from active cells (primarily pyramidal cells (Fig. 2), though interneuron potentials (Fig. 3) were used for cross-frequency coupling comparison - explained below), and a local field potential (LFP) signal obtained from a point equidistant from all of the cells. Overall, in order to test our hypothesis, we ran three different scenarios on the model. In one scenario, the efficacy of $\mathrm{Na}^{+}-\mathrm{K}^{+}$ATPase pump was adjusted down from $100 \%-$ this was accomplished by applying a factor $\mathrm{f}$ to equation (2) with $f$ ranging from 1.0 to 0.55 (specifically the following values - 1.0, 0.9, 0.7, 0.6, 0.55). In another, the rate of glial potassium clearance was reduced (by 10\%, 25\%, 45\%, and 
TABLE III

BREAKDOWN OF THE MAIN PARAMETERS AND INITIAL VALUES USED IN THE MODEL

\begin{tabular}{|c|c|c|c|c|c|}
\hline Parameter & Value & Description & Parameter & Value & Description \\
\hline$P Y: g_{K, d r}$ & $200 \mathrm{mS} / \mathrm{cm}^{2}$ & $\begin{array}{c}\text { Somatic pyramidal cell } \mathrm{K}_{\mathrm{DR}} \text { channel } \\
\text { maximal conductance }\end{array}$ & $P Y: g_{\text {leak }}$ & $0.03 \mathrm{mS} / \mathrm{cm}^{2}$ & $\begin{array}{c}\text { Somatic pyramidal cell leak channel } \\
\text { maximal conductance }\end{array}$ \\
\hline$P Y: g_{K, a}$ & $0.01 \mathrm{mS} / \mathrm{cm}^{2}$ & $\begin{array}{l}\text { Somatic pyramidal cell } \mathrm{K}_{\mathrm{A}} \text { channel } \\
\text { maximal conductance }\end{array}$ & $I N: g_{\text {leak }}$ & $0.03 \mathrm{mS} / \mathrm{cm}^{2}$ & $\begin{array}{l}\text { Somatic interneuron leak channel } \\
\text { maximal conductance }\end{array}$ \\
\hline$P Y: g_{K, c}$ & $2.5 \mathrm{mS} / \mathrm{cm}^{2}$ & $\begin{array}{c}\text { Somatic pyramidal cell } \mathrm{K}_{\mathrm{C}} \text { channel } \\
\text { maximal conductance }\end{array}$ & $I N: g_{K, d r}$ & $200 \mathrm{mS} / \mathrm{cm}^{2}$ & $\begin{array}{l}\text { Somatic interneuron } \mathrm{K}_{\mathrm{DR}} \text { channel } \\
\text { maximal conductance }\end{array}$ \\
\hline$I N: g_{K, a h p}$ & $0.1 \mathrm{mS} / \mathrm{cm}^{2}$ & $\begin{array}{l}\text { Somatic pyramidal cell } \mathrm{K}_{\mathrm{AHP}} \text { channel } \\
\text { maximal conductance }\end{array}$ & $P Y: g_{K, a h p}$ & $0.1 \mathrm{mS} / \mathrm{cm}^{2}$ & $\begin{array}{l}\text { Somatic interneuron } \mathrm{K}_{\mathrm{AHP}} \text { channel } \\
\text { maximal conductance }\end{array}$ \\
\hline$P Y: g_{N a}$ & $3450 \mathrm{mS} / \mathrm{cm}^{2}$ & $\begin{array}{l}\text { Somatic pyramidal cell } \mathrm{Na} \text { channel } \\
\text { maximal conductance }\end{array}$ & $I N: g_{N a}$ & $3800 \mathrm{mS} / \mathrm{cm}^{2}$ & $\begin{array}{l}\text { Somatic interneuron } \mathrm{Na} \text { channel } \\
\text { maximal conductance }\end{array}$ \\
\hline$P Y: g_{N a, p}$ & $3.5 \mathrm{mS} / \mathrm{cm}^{2}$ & $\begin{array}{l}\text { Somatic pyramidal cell } \mathrm{Na}_{\mathrm{p}} \text { channel } \\
\text { maximal conductance }\end{array}$ & $\boldsymbol{K}_{\alpha}$ & $2.5 \mathrm{mM}$ & $\begin{array}{l}\text { Potassium-related constant for } \\
\text { sodium-potassium pump }\end{array}$ \\
\hline$P Y: g_{C a}$ & $0.0165 \mathrm{mS} / \mathrm{cm}^{2}$ & $\begin{array}{l}\text { Somatic pyramidal cell Ca channel } \\
\text { maximal conductance }\end{array}$ & $N a_{\alpha}$ & $20 \mathrm{mM}$ & $\begin{array}{l}\text { Sodium-related constant for } \\
\text { sodium-potassium pump }\end{array}$ \\
\hline$C_{m}$ & $\mu \mathrm{F} / \mathrm{cm}^{2}$ & Membrane capacitance & $P Y: V_{m}$ & $-75 \mathrm{mV}$ & Pyramidal cell membrane voltage \\
\hline$I_{\max }$ & $20 \mathrm{~mA} / \mathrm{cm}^{2}$ & $\begin{array}{l}\text { Maximum sodium-potassium pump } \\
\text { current }\end{array}$ & $I N: V_{m}$ & $-85 \mathrm{mV}$ & Interneuron membrane voltage \\
\hline$[B]_{T}$ & $500 \mathrm{mM}$ & Total free buffer & Glia: $V_{m}$ & $-85 \mathrm{mV}$ & Glial membrane voltage \\
\hline$[K]_{i} /[K]_{o}$ & $130 / 4 \mathrm{mM}$ & $\begin{array}{c}\text { Intra/extracellular potassium } \\
\text { concentration }\end{array}$ & {$[K]_{g}$} & $100 \mathrm{mM}$ & Glial potassium concentration \\
\hline$[\mathrm{Na}]_{i} /[\mathrm{Na}]_{o}$ & $10 / 145 \mathrm{mM}$ & Intra/extracellular sodium concentration & {$[\mathrm{Na}]_{\mathrm{g}}$} & $15 \mathrm{mM}$ & Glial sodium concentration \\
\hline$[K]_{T H}$ & $10 \mathrm{mM}$ & Activation threshold for glial buffer & $g_{A M P A}$ & $9 / 3 \mathrm{nS}$ & $\begin{array}{l}\text { AMPA conductances (PY-PY and } \\
\text { PY-IN) }\end{array}$ \\
\hline$g_{N M D A}$ & $0.9 / 0.3 \mathrm{nS}$ & $\begin{array}{l}\text { NMDA conductances (PY-PY and } \\
\text { PY-IN) }\end{array}$ & $g_{G A B A}$ & $9 \mathrm{nS}$ & Inhibitory GABA conductance \\
\hline
\end{tabular}

$50 \%$ ), whereas in the third the expression of $\mathrm{K}_{\mathrm{AHP}}$ was scaled down by reducing the maximal conductivity (specifically by reducing it by $10 \%, 30 \%, 40 \%$, and $45 \%$ ). In order to obtain LFP data, the following equation was used on transmembrane currents:

$$
V_{L F P}=\frac{1}{4 \pi \sigma} \sum_{i}^{n} \sum_{j}^{m} \frac{I_{i j}}{\left|r_{i j}\right|}
$$

where $\sigma$ is the conductivity of the medium, $n$ is the total number of neuronal compartments across all neurons, $m$ is the number of transmembrane currents for each neuron, $I_{i j}$ is the $\mathrm{j}^{\text {th }}$ transmembrane current of the $\mathrm{i}^{\text {th }}$ neuronal compartment and $r_{i j}$ is the distance from that current to the point of LFP computation [29].

Before analyzing the data, we worked to establish reliable baseline readings with which to compare our results. One such baseline was the output of the model in a resting state (trace (i) in Fig. 2). We classified the state as resting if the transmembrane potential and the LFP predominantly oscillated around the resting potential and the occasional spike was shorter than $80 \mathrm{~ms}$ in duration. This baseline was used as a benchmark for energy present in the network. Another baseline was the hyperexcitable state (trace (ii) in Fig. 2), which was achieved by decreasing all of the hyperexcitability pathways by $10 \%$ simultaneously. Hyperexcitable state was distinguished from an occasional individual spike that can appear in a resting state using the following criteria: the average discharge duration had to be at least $80 \mathrm{~ms}$ and discharges had to occur with frequency of at least $2 \mathrm{~Hz}$. The state in trace (ii) in Fig. 2 was the baseline for the discharge duration.

During the study we generated several runs of 30 seconds of model output for each state. After making sure that the features we observed are repeated in multiple runs, we subdivided the data into 10 second segments for analysis. Data was then analyzed in two different ways - by measuring the level of excitability of the model and by evaluating cross-frequency coupling dynamics produced by it.

In order to analyze the level of model excitability, we used several approaches to approximate the amount of energy present in the network. To find the optimal way of measurement, three different methods were initially evaluated using the squared value of the signal $\left(\mathrm{x}^{2}\right)$, the Teager energy operator (a measure sometimes used in quantifying the energy of excitable neural networks [32]), and the technique we called the kernel convolution method. The Teager energy operator was used in its discrete form as follows:

$$
T E O=(|x[n]|)^{2}-x[n+1] * x[n-1]
$$

The kernel convolution method of estimating the energy of the network is achieved by convolving the squared trace with the following function:

$$
H=\beta t * \exp (-\beta t)
$$

where $\beta$ is the parameter that controls the decay rate of the kernel mode response and was chosen to be $2 \mathrm{~Hz}$ (units are chosen such that $H$ function is unitless) which 
A
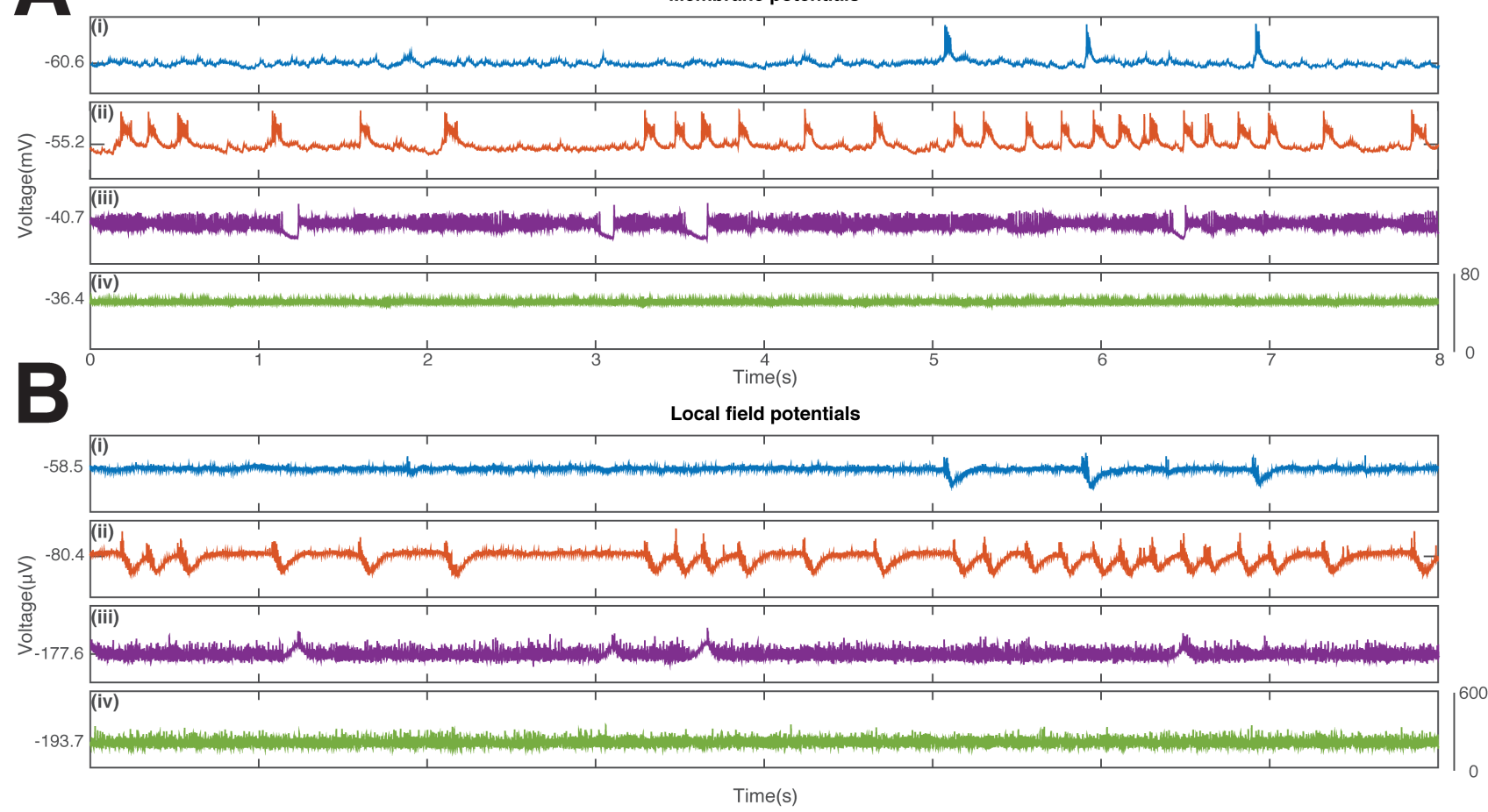

Fig. 2. A) Comparison between resting state, which was used as a baseline for energy calculation (i), hyperexcitable state which was used as a baseline for SED duration comparison (ii), long duration SED (iii) and continuous depolarization (iv) states using transmembrane voltage recorded from one pyramidal cell somatic compartment. B) The same comparison as in A), but for the network response recorded as the local field potential equidistant from all cell models For each subfigure, all of the traces are plotted on the same scale (the scale is shown in the bottom right corner), so as the duration of the spontaneous discharge increases, the amplitude decreases, whereas the average potential either continuously increases (for part A) or decreases (for part B) - both findings consistent both with physiological observations (Fig. 1 in [30]) and other computational results (Fig. 1 in [31]). In this case the sample transition is shown, achieved by manipulating extracellular potassium clearance rate. On the y-axis, average value, or baseline, of the 30-second generated signal is shown.

was consistent with measurements made in low- $\mathrm{Mg}^{2+} / \mathrm{high}-$ $\mathrm{K}^{+}$models of epilepsy [20], [33]. This has been suggested to
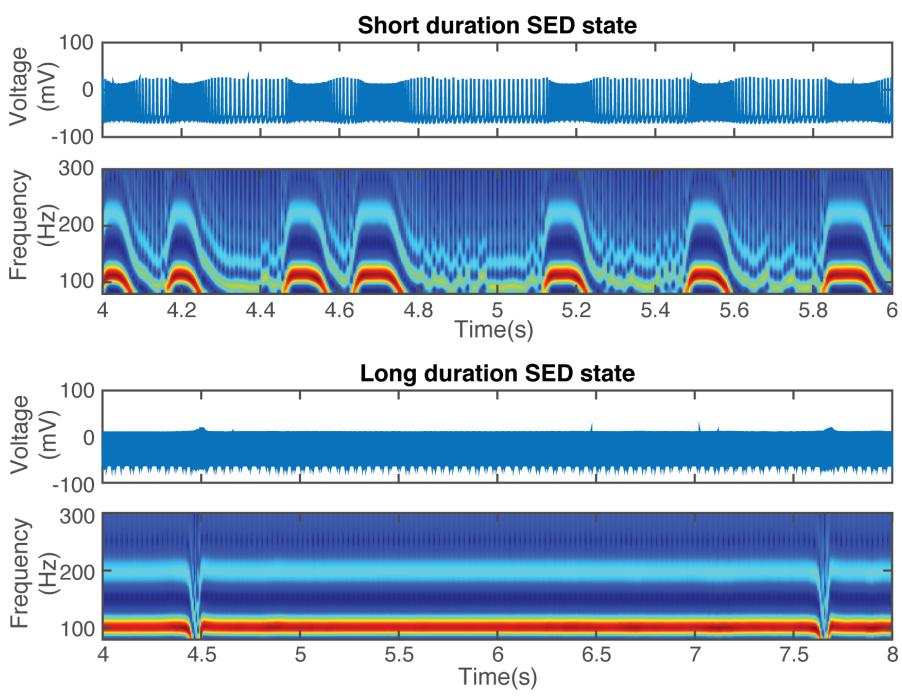

Fig. 3. Interneuronal transmembrane potential during a short duration SED and long duration SED states, achieved by manipulating extracellular potassium clearance rate. Below each trace, dominant frequencies in the high $(80-300 \mathrm{~Hz})$ frequency bands are shown, with red being the incidence of highest power. Low frequencies are not shown, as they were found to be largely absent. be a good approximation of the Teager energy measurement while significantly reducing computational requirements [34], and we wanted to perform the validation of this approach. We also compared these measures to the average voltage of the model output for each state. Five-second windows of resultant energy traces were integrated using Simpson's rule to obtain an average reading irrespective of the trace duration:

$$
\int_{a}^{b} f(x) \approx \frac{b-a}{6}\left(f(a)+4 f\left(\frac{a+b}{2}\right)+f(b)\right)
$$

Simpson's rule was used since usually it is a better approximation of a non-linear curve than simpler methods (for example MATLAB's trapezoidal method). This average energy level was then normalized to the model resting state and compared across different pathways, levels of impairment, and duration of the SEDs. In order to streamline the calculation of SED duration, the membrane potential trace was bandpass filtered at 6-10 Hz to extract the envelope of SEDs [35]. Filtered trace was then convolved with a second Gaussian derivative to find the inflexion points in the envelope, which correlate with SED onset/termination [36].

In order to determine the cross-frequency coupling dynamics between LFOs and HFOs, index of cross-frequency coupling $\left(\mathrm{I}_{\mathrm{CFC}}\right)$ was computed, using Tort's method [37]. Briefly, the $\mathrm{I}_{\mathrm{CFC}}$ was found by removing the mean of the original signal and decomposing it into two - high frequency 

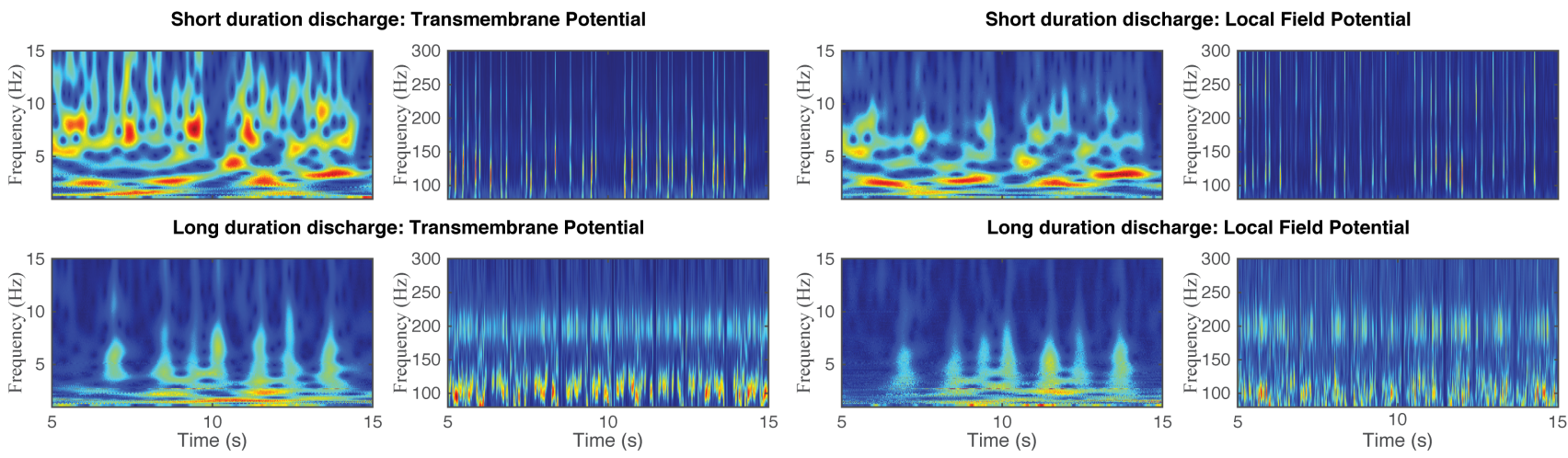

Fig. 4. Dominant frequencies in the low $(0.5-15 \mathrm{~Hz})$ and high $(80-300 \mathrm{~Hz})$ frequency bands in a pyramidal cell transmembrane potential and LFP traces in two different states - short duration SED, and long duration SED.

component (in this study, 80-400 Hz), and low frequency component $(1-12 \mathrm{~Hz})$ - using continuous wavelet transform (CWT; complex Morlet wavelet with $0.8125 \mathrm{~Hz}$ center frequency and $5 \mathrm{~Hz}$ bandwidth was used for CWT, following a common set up used in our lab [3]). The amplitude envelope was then extracted from the high frequency component, denoted $A\left(\hat{t}, f_{H}\right)$. Similarly, the phase was extracted from the low frequency component of the original signal, denoted $\varphi\left(\hat{t}, f_{H}\right)$. Finally, the phase signal is binned into $20^{\circ}$ windows and the average amplitude value over that window is found making it the $\mathrm{I}_{\mathrm{CFC}}$ shown in comodulograms. All of the data analysis was done in MATLAB.

\section{RESULTS}

After establishing appropriate benchmarks, three progressions were done for each pathway involved in generating a hyperexcitable model. Fig. 2 shows two major comparative states that were achieved (in the case of Fig. 2, through making adjustments to the glial potassium clearance pathway) - a state that is dominated by long duration discharges (over 2 seconds long) shown in trace (iii) in both Fig. 2A and Fig. 2B; as well as a state of continuous depolarization that lasts until the end of the simulation - trace (iv) in Fig. 2. In both cases, as the duration of the discharge increased, the amplitude of potential fluctuations decreased; furthermore, the average potential of the signal changed as the discharge duration increased - the average became more depolarized in the case of transmembrane voltage (Fig. 2A), but becomes more negative when shown as the LFP (Fig. 2B). Finally, the frequency of discharges also decreases and interesting crossfrequency coupling features arise as seen in the next section.

It is useful to compare pyramidal cell transmembrane potential and LFP with the interneuronal transmembrane potential. Fig. 3 shows the membrane voltage of a sample interneuron - the interneuron is fast spiking and has more recurrent and regular spikes compared to the pyramidal cell. Furthermore, Fig. 3 shows the predominance of $100 \mathrm{~Hz}$ and $200 \mathrm{~Hz}$ frequencies in interneurons both during short and long duration SED state (low frequencies are not shown for interneuron, since they were found to be largely absent). This can be contrasted with the dominant frequencies in the sample pyramidal cell and LFP (Fig. 4) for both the short and the long duration
SED states, where there is a dominance of $3-9 \mathrm{~Hz}$ frequencies during short duration SEDs and 1-5 Hz, as well as some high frequencies, during long duration SEDs.

Both Fig. 5 and Fig. 6 show comparison between the measure of cross-frequency coupling derived from the LFP trace and the CFC measures obtained from individual transmembrane voltage traces - for short duration and long duration SED states respectively (obtained by reduction of glial potassium clearance pathway). In case of pyramidal cells, $\mathrm{I}_{\mathrm{CFC}}$ is dominated by extremely high frequency $(300+\mathrm{Hz})$ modulated by theta $(6-8 \mathrm{~Hz})$, whereas that is not the case for either interneuronal or LFP-derived I $\mathrm{C}_{\mathrm{CFC}}$ 's. In Fig. 6 pyramidal cell $\mathrm{I}_{\mathrm{CFC}}$ highlight similar frequencies as the LFP one - the deltaHFO coupling without any artifact due to spikes. By contrast, interneurons demonstrate dynamics that are quite different from that - with several very narrow CFC bands, centered around theta frequencies.

The average SED duration and energy of the networks for each parameter manipulation is shown in table IV, where the durations are split between the averages for short duration SEDs (defined as under 2 seconds long), and averages for long duration SEDs. The distribution of SED durations and interSED durations for both short duration SED and long duration SED states are also shown in Fig. 7 with the gamma fit overlaid over the histograms.

The results of this study are further summarized in Fig. 8 . First, the level of excitability change in the model is compared across three different pathways (Fig. 8A). Network excitability was estimated through the kernel convolution energy measure, and in all three cases there is a similar trend towards the higher hyperexcitability with further pathway reduction. The duration of an SED is related to the level of excitability of the system (Fig. 8B) independent of the pathway that caused that excitability. The figure shows that there is an exponential relationship between the excitability percent change and SED duration (normalized with respect to the hyperexcitable baseline state). Long duration SED could be said to occur somewhere between $800 \%$ and $900 \%$ change in excitability of the system, with continuous depolarization state being beyond the $900 \%$ bound (not shown in the figure). In Fig. 8C we tracked the change in average extracellular potassium concentration during different states, to see how parameter changes 


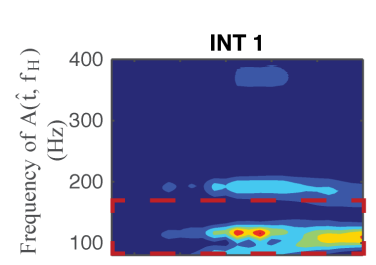

INT 2

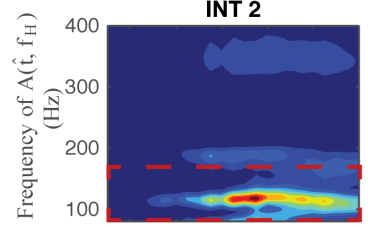

INT 3

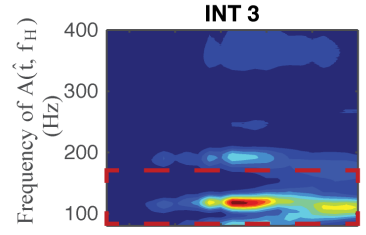

INT 4

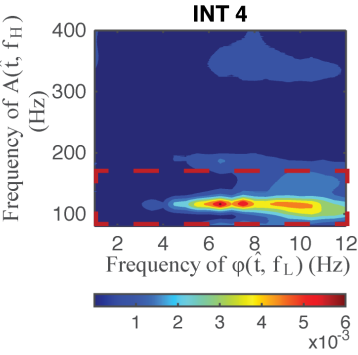

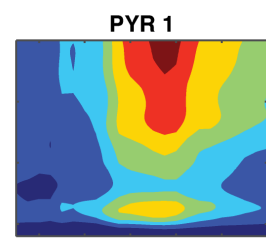

PYR 2

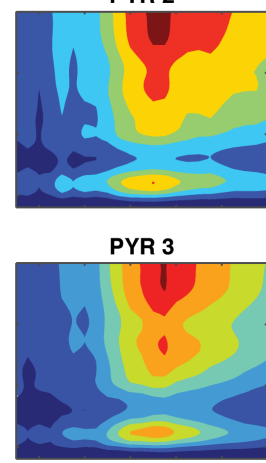

PYR 4

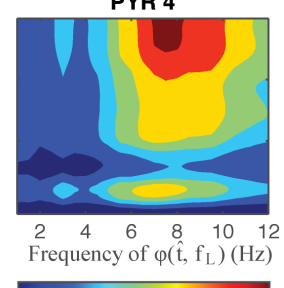

$0.020 .040 .060 .08 \quad 0.10 .1$

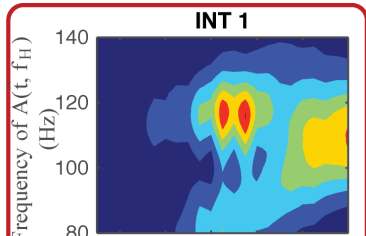

INT 2

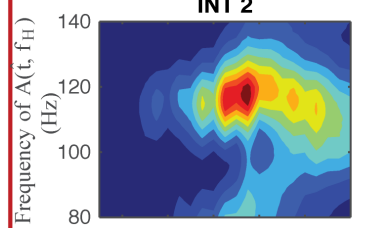

INT 3

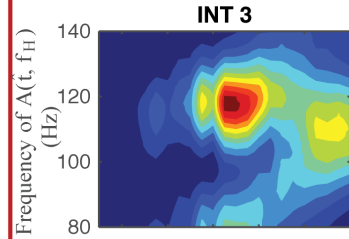

INT 4

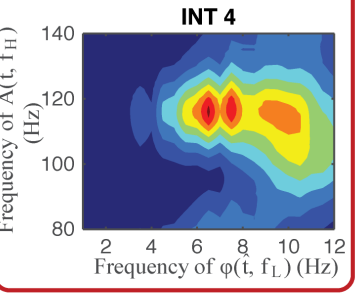

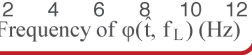

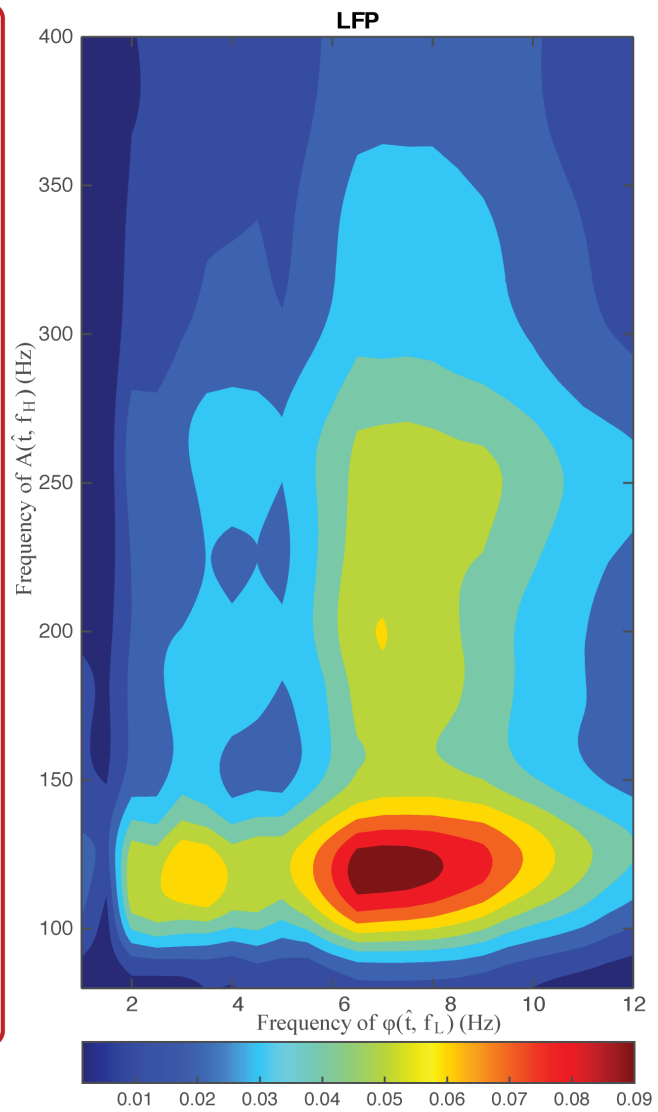

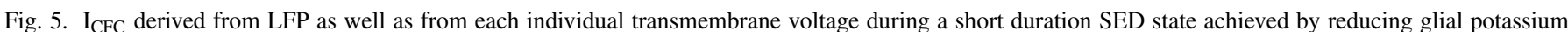
clearance pathway. The inset in the red box shows the close-up for the interneuronal-derived comodulograms. Note that both the inset and the pyramidal cell $\mathrm{I}_{\mathrm{CFC}}$ show theta-HFO (100-150 Hz) CFC, which is also seen in the LFP-derived comodulogram. However, in pyramidal cell $\mathrm{I}_{\mathrm{CFC}}$ theta-HFO CFC is overshadowed by higher frequencies $(300+\mathrm{Hz})$ - likely an artifact due to spiking. Also note that although there are $150-200 \mathrm{~Hz}$ frequencies present in interneuronal transmembrane potential (Fig. 3), they do not show up in the $\mathrm{I}_{\mathrm{CFC}}$.

affected extracellular potassium. With minor variations, the main observation links dysfunction in the proposed pathways to the rise in extracellular potassium. We also wanted to see how the choice of energy method can influence excitability measurement in Fig. 8A and Fig. 8B. Fig. 8D shows how other energy measures $\left(\mathrm{x}^{2}\right.$, Teager energy, and the average potential) compare to the kernel convolution energy estimation method. For each method, Pearson coefficient was computed and both $\mathrm{x}^{2}$ and average potential energy measures were found to have the coefficient close to 1; whereas Teager energy measure showed worse correlation with the kernel convolution.

\section{Discussion}

This study aims to evaluate the relationship between excitability of the network, SED duration, and cross-frequency coupling features. Transmembrane voltage and LFP changed as a function of network parameters (Fig. 2) as follows i) the duration of discharges increased, ii) the amplitude of potential fluctuations around the mean depolarization potential decreased, and iii) the apparent frequency of discharge occurrence decreased. These findings were consistent with physiological observations of seizure recordings [30], which was a good validation of the model. Furthermore, the changes observed in the pyramidal cell transmembrane potential discharges were comparable both in shape and duration with in vivo recordings obtained from rodent models (Fig. 3 in [38]). Furthermore, our group has reported that in animal models of Rett's syndrome short duration SEDs were associated with non-life-threatening conditions whereas longer duration SEDs showed worse clinical outcomes, and the improvement in the prognosis was concurrent with their decline [2]. Also, another recent study compared psychogenic non-epileptic seizures (PNES) with epileptic seizures (ES) in patients found that longer duration discharges were associated with ES alone, whereas less severe combined ES and psychogenic seizures were of shorter durations [39]. These results suggest a link between excitability, symptom severity, and SED duration, that we explore further in our study.

SED duration distribution changed from short to long duration SED states with both the average and the spread of durations increasing, whereas the inter-SED durations stayed relatively the same in both cases (Fig. 7). Following the work of Bauer et al. [40], we also used the data to obtain a gamma distribution fit which showed that short SED durations had a distribution with $\alpha>1$ (6.3, Confidence Interval: 5.3-7.4), which is consistent with the analysis done in [35] on the in vivo data. Long SED durations had a gamma distribution with 

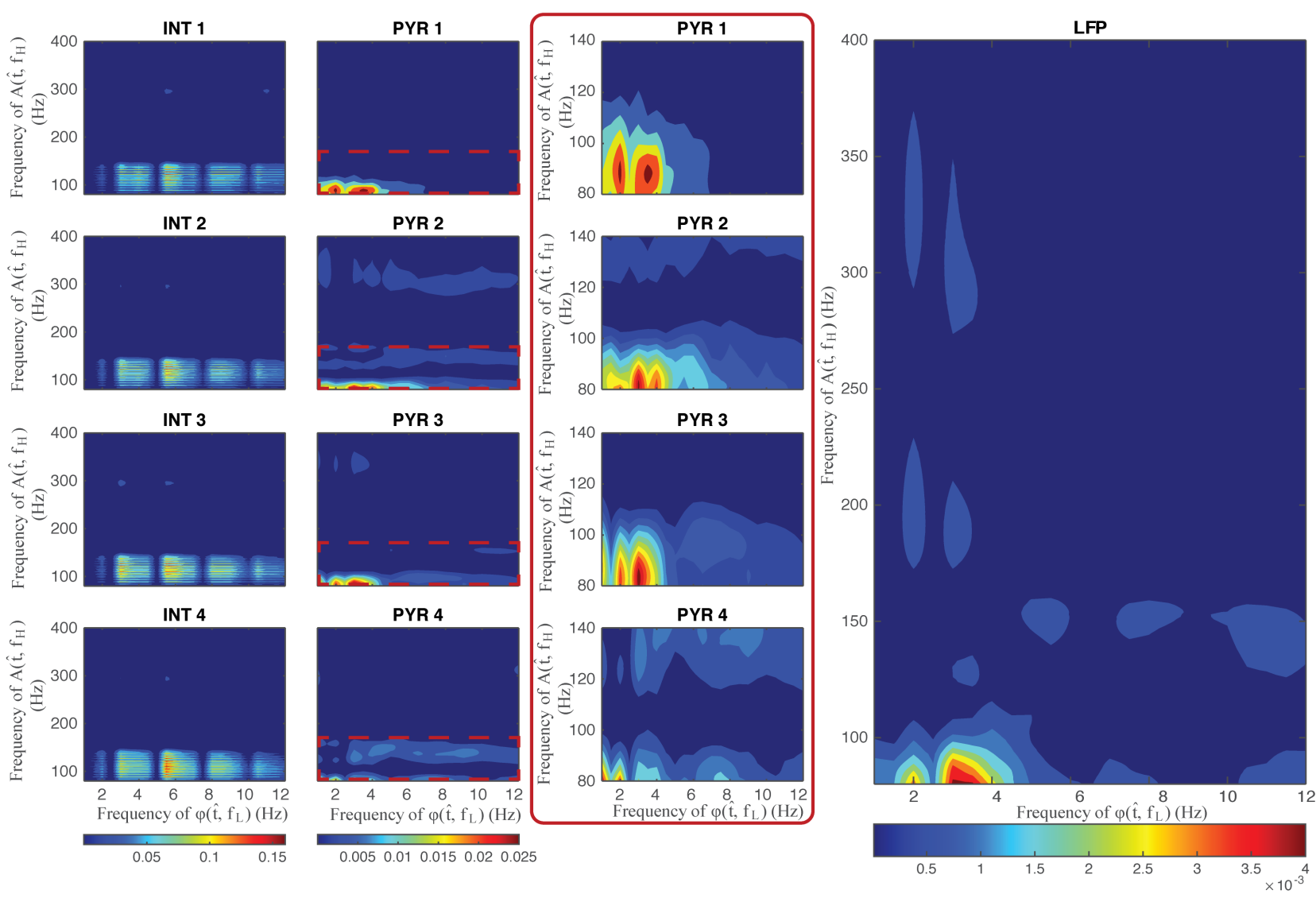

Fig. 6. $\mathrm{I}_{\mathrm{CFC}}$ derived from LFP as well as from each individual transmembrane recording during a long duration discharge state achieved by reducing glial potassium clearance pathway. The inset in the red box shows a close-up of pyramidal cell CFC. Again, note that although interneurons demonstrate strong $200 \mathrm{~Hz}$ frequencies (Fig. 3), they don't show up in the $\mathrm{I}_{\mathrm{CFC}}$.

$\alpha<1$ (0.57, CI:0.34-0.96), suggesting that although short duration SED state can be predicted, long durations occur at random [41]. In both cases inter-SED duration were Poisson distributed ( $\alpha \approx 1$, with CI: 0.84-1.5), which is similar to the distribution at which action potentials occur [22].

Fig. 5 and Fig. 6 show that dominant low frequencies involved in CFC changed from theta $(4-8 \mathrm{~Hz})$ to delta (1-4 $\mathrm{Hz})$ frequency bands during the transition from short duration to long duration SED state; a finding that is similar to iEEG analysis done by our group previously [3]. These figures also suggest that the main contributor to cross-frequency coupling derived from LFP are pyramidal cells, as their dynamics seem to mimic the dynamics of LFP CFC. In Fig. 3 and Fig. 4, the dominant frequencies in the LFP signal match closely those in the pyramidal cell transmembrane potential signal but not in the interneuron, especially during the short duration SED state. Additionally, comparing Fig. 3 and Fig. 4 with detailed $\mathrm{I}_{\mathrm{CFC}}$ 's of Fig. 5 and Fig. 6 shows that CFC does not always involve dominant frequencies. This point was investigated in an iEEG analysis study [3], where it was determined that CFC, and not individual frequencies, can act as a reliable biomarker.

We performed both phase and amplitude shuffling to produce surrogate data to analyze the significance of our CFC findings. In both cases, the $\mathrm{I}_{\mathrm{CFC}}$ obtained from original data was significantly different (within at least a 95\% confidence interval) from the generated surrogate data $(\mathrm{N}=200)$. Overall, CFC features that we have discovered in this study closely mirror the phenomena found in our team's measurements both in epileptic patients [3] and rodent models [2]. Moreover, a CFC signal obtained from the scalp EEG of the same epileptic patients as in [3] was used for source imaging of the epileptogenic zones [42]. On one hand this acts as a further validation of our model; whereas on the other hand it suggests potential cellular pathways that generate these CFC phenomena.

To further understand hyperexcitability of neuro-glial networks we measured the energy of the network. As network parameters changed, it transitioned to states with high energy in a path independent manner (Fig. 8A). Although the rate of energy (and consequently excitability of the network) increase is different between different pathways, the overall result is the same - a steady increase of network energy with the increase of parameter change. It could be noted that $\mathrm{Na}^{+}-\mathrm{K}^{+}$ATPase pump appears to have the most effect on the excitability, whereas the potassium clearance rate is less effective. However, since the clearance rate is a rough phenomenological approximation using buffering model, that might be due to the approximation and not potassium dynamics. Path independence of excitability change is further investigated in Fig. 8C, where the change in average extracellular potassium concentration is tracked as 
the network transitions between short and long duration SED states. Overall the results suggest that multiple pathways that can lead to heightened excitability of the network, which is consistent with the view of biological networks suggested by Marder et al, 2013 [43]. The comparison of different energy measures (Fig. 8D) shows that both $\mathrm{x}^{2}$ and average potential energy measures are close in excitability estimation to the kernel convolution, with Pearson coefficients close to 1 . In contrast, Teager energy operator is worse at estimating network excitability - especially at higher excitability levels. Although $\mathrm{x}^{2}$ is a commonly used energy measure, the benefit of using kernel convolution is two fold - i) its parameters are tuned specifically for the hyperexcitable brain networks, obtained from empirical data by our lab [33], ii) the kernel represents the dominant neuronal mode, which links excitability to the modes present in the brains networks [44]. High correlation between $\mathrm{x}^{2}$ and the kernel convolution method (as seen in Pearson coefficient of 1) further reinforces the applicability of latter energy measurement method. Furthermore, the comparison average LFP measure and $\mathrm{x}^{2}$ and kernel convolution methods highlights the observation that with the increase in excitability, the duration of depolarization increases and the mean potential during the depolarization changes. As this is the model, we do not expect to see any artifactual change in the baseline potential, and so any change in the mean can be attributed to the cell being more depolarized due to higher excitability. Even in the continuous depolarization state, as seen in Fig. 2 (trace (iv)), there is still a large amount of activity going on both in the LFP and transmembrane potential traces.

The relationship between the energy of the state and the average duration of SEDs is shown in Fig. 8B, where the average duration of SEDs depends on the level of excitability of the network in an exponential manner irrespective of the
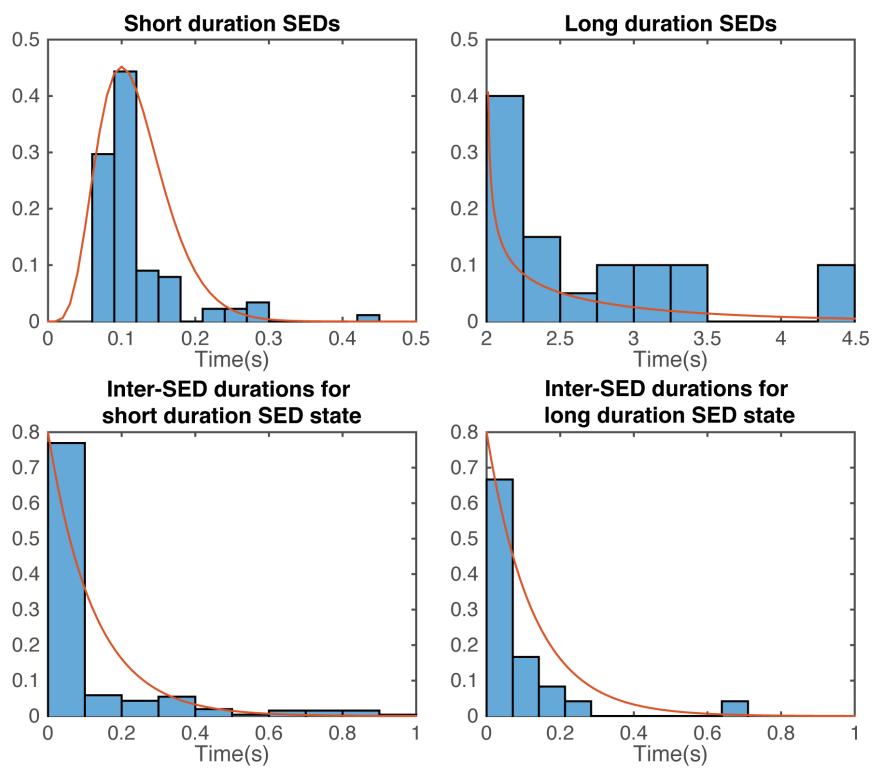

Fig. 7. SED duration and inter-SED duration distributions for short duration SED states and long duration SED states. For short duration SEDs, $\alpha=6.28$; long duration SEDs, $\alpha=0.57$; inter-SED $\alpha \approx 1$.
TABLE IV

AVERAGE DURATION OF THE SHORT AND LONG DURATION SEDS FOR EACH PARAMETER ADJUSTMENT, AS WELL AS THE RELATIVE ENERGY VALUE $^{1}$

\begin{tabular}{|c|c|c|c|c|c|}
\hline \multicolumn{3}{|c|}{ Network Parameters } & \multicolumn{2}{|c|}{ Duration (s) } & \multirow{2}{*}{$\begin{array}{c}\text { Relative Energy } \\
\text { of Network }\end{array}$} \\
\hline $\mathbf{K}_{\mathrm{AHP}}$ & $\mathbf{K}_{\text {rate }}$ & $\mathbf{I}_{\text {pump }}$ & Short & Long & \\
\hline $0 \%$ & $0 \%$ & 1 & - & - & $1 *$ \\
\hline $10 \%$ & $10 \%$ & 0.9 & $0.08 \pm 0.02$ & - & 3.5 \\
\hline $30 \%$ & $10 \%$ & 0.9 & $0.1 \pm 0.01$ & - & 8.0 \\
\hline $40 \%$ & $10 \%$ & 0.9 & $1.6 \pm 0.3$ & $3.7_{ \pm 0.7}$ & 10.8 \\
\hline $45 \%$ & $10 \%$ & 0.9 & - & eos & 13.2 \\
\hline $10 \%$ & $35 \%$ & 0.9 & $0.1 \pm 0.02$ & - & 4.3 \\
\hline $10 \%$ & $45 \%$ & 0.9 & $1.6 \pm 0.2$ & $2.6 \pm 0.5$ & 10.7 \\
\hline $10 \%$ & $50 \%$ & 0.9 & - & eos & 13.1 \\
\hline $10 \%$ & $10 \%$ & 0.7 & $0.2 \pm 0.08$ & - & 10.0 \\
\hline $10 \%$ & $10 \%$ & 0.6 & $1.9_{ \pm 0.1}$ & $2.9_{ \pm 0.7}$ & 13.0 \\
\hline $10 \%$ & $10 \%$ & 0.55 & - & eos & 15.8 \\
\hline
\end{tabular}

maneuver used. Due to the exponential relationship between SED duration and network excitability, long duration SEDs occur between roughly $800 \%$ and $900 \%$ increase in excitability, whereas short duration SEDs occur below that level of excitability. The Pearson coefficient between the measured network energy and SED of 0.8 suggests an association between SED durations and excitability. However, the exponential function had the highest goodness of fit $\left(R^{2}=0.83\right)$, compared to that of the linear fit $\left(R^{2}=0.7\right)$.

To account for glial influence on network hyperexcitability, we incorporated astrocytes into the model in two major ways. The first was glial potassium clearance (equation (12)), which was one of the parameters we varied in the network to achieve excitability. When we set potassium clearance to effectively be zero, the model entered a highly hyperexcitable state similar to trace (iv) in Fig. 2. Astrocytes also maintained excitability (to the lesser extent) through glial $\mathrm{Na}^{+}-\mathrm{K}^{+}$ATPase pump, which contributed to balancing of extracellular ionic concentrations. Reducing or removing that term produced results similar to (though lesser) the blue trace in Fig. 8A. These results, then, show the invaluable part that astrocytes play in maintaining network excitability.

Although our focus on the common pathways of hyperexcitability provided us with a reasonable scope for the study, several areas of potential influence on the excitability of the system (and consequently duration of an SED) could be investigated. First, there has been some research into metabolic effects of hyperexcitability, such as the role of glutamate in generating SEDs [45], which would focus on excitatory glutamatergic receptors and synaptic (rather than intrinsically neuro-glial) effects of hyperexcitability. Another factor is the osmosis-driven potassium concentration increase due to volume changes both in astrocytes and extracellular space. Some research indicates that volume changes due to

\footnotetext{
${ }^{1} \mathbf{K}_{\mathrm{AHP}}$ refers to adjustments to the potassium afterhyperpolarization channel, $\mathbf{K}_{\text {rate }}$ - potassium clearance rate, and $\mathbf{I}_{\text {pump }}$ - efficacy of the $\mathrm{Na}^{+}-\mathrm{K}^{+}$ ATPase pump. ${ }^{*}$ Energy value is shown as relative to the resting state, thus the first row has it as 1. "eos" refers to a depolarization lasting for the entirety of the simulation time (even when the simulation time is doubled).
} 

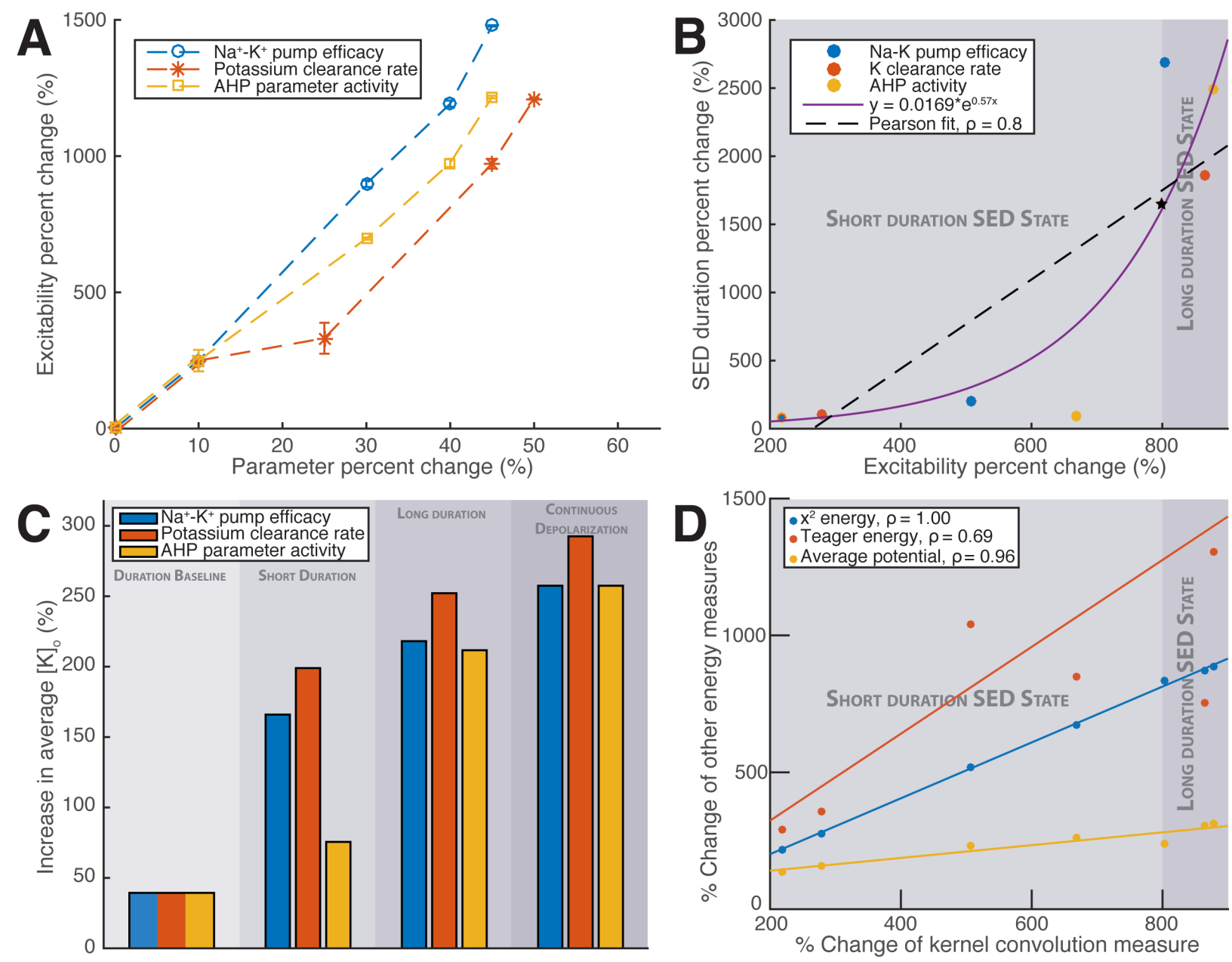

Fig. 8. Relationship between parameter changes, excitability and SED duration. A) All three pathways cause an increase in excitability of the network, with $\mathrm{Na}^{+}-\mathrm{K}^{+}$ATPase pump being the most effective. Vertical bars show standard error. B) SED duration depends on the level of excitability of the system independent of the pathway that has caused it, with an exponential relationship $y=0.0169 e^{0.57 x}\left(R^{2}=0.83\right)$. Beyond about $900 \%$ change in excitability from resting baseline, the system enters continuous depolarization state. The larger point shows the hyperexcitable baseline state, and black star highlights the point at which the transition from short duration to long duration SED happens (i.e. average $2 \mathrm{~s}$ duration SED). The dashed black line shows the linear fit $\left(R^{2}=0.7\right)$ corresponding to the calculated Pearson coefficient (0.8). C) During parameter changes for all three pathways, extracellular potassium concentration increased, with potassium clearance rate causing the most increase. D) Comparison of energy measure $\left(\mathrm{x}^{2}\right.$, Teager energy, average potential) performance with the performance of the kernel convolution method, with Pearson coefficients listed in the legend. Note: since the duration of the discharge was not measured in the continuous depolarization state, those points were not used in Fig. 8B.

increase in blood circulation could result in the increase of system excitability [46]. Finally, although in this model we included some gap junctions between pyramidal cells and interneurons, many more exist between many cells in the brain, including interneurons, pyramidal cells, and astrocytes, which can have varying effect on both potassium concentrations and epileptogenesis [47]. Our preliminary results on effects of gap junctions in neuro-glial networks have shown that astrocytic gap junctions could help to ameliorate seizure-like states [48], possibly due to increased potassium clearance. Including a more diverse selection of gap junctions in the model could help model the additional role of astrocytes in maintaining excitability balance in neuro-glial networks.

\section{CONCLUSION}

In this study we focused on the commonly proposed pathways of generating hyperexcitable conditions in neuro-glial networks $-\mathrm{Na}^{+}-\mathrm{K}^{+}$ATPase pump, glial potassium clearance, and potassium afterhyperpolarization channel. First, the the model identified the transition from short duration SED to long duration SED states as excitability level of the network increased, as well as what factors cause the increase in excitability. Furthermore, the change in SED states seems to be largely independent of the parameter being changed, which is consistent with other research into the origins of rhythmic oscillations [43]. Second, model highlighted the importance of glial factors in hyperexcitability - namely potassium clearance and $\mathrm{Na}^{+}-\mathrm{K}^{+}$ATPase pump. Third, model findings suggested biophysical basis for cross-frequency coupling that was observed in epileptic patients [3] and rodent models [2]. The dysfunction in the three pathways lead to the dominance of delta-HFO cross-frequency coupling, a feature which could be used as a biomarker for hyperexcitable neuro-glial networks of the brain. 


\section{REFERENCES}

[1] R. A. B. Badawy, A. S. Harvey, and R. A. L. Macdonell, "Cortical hyperexcitability and epileptogenesis: Understanding the mechanisms of epilepsy - Part 1," J. Clin. Neurosci., vol. 16, no. 4, pp. 355-365, 2009.

[2] M. Lang et al, "Rescue of behavioral and EEG deficits in male and female Mecp2-deficient mice by delayed Mecp2 gene reactivation," Hum. Mol. Genet., vol. 23, no. 2, pp. 303-318, 2014.

[3] M. Guirgis, et al, "Defining regions of interest using cross-frequency coupling in extratemporal lobe epilepsy patients," J. Neural Eng., vol. 12, no. 2, p. 26011, 2015.

[4] J. Lisman, "The theta/gamma discrete phase code occuring during the hippocampal phase precession may be a more general brain coding scheme," Hippocampus, vol. 15, pp. 913-922, 2005.

[5] G. Seifert, G. Carmignoto, and C. Steinhuser, "Astrocyte dysfunction in epilepsy," Brain Res. Rev., vol. 63, no. 1-2, pp. 212-221, 2010.

[6] L. E. Clarke and B. A. Barres, "Emerging roles of astrocytes in neural circuit development.," Nat. Rev. Neurosci., vol. 14, no. 5, pp. 311-21, 2013.

[7] G. G. Somjen, H. Kager, and W. J. Wadman, "Computer simulations of neuron-glia interactions mediated by ion flux," J. Comput. Neurosci., vol. 25, no. 2, pp. 349-365, 2008.

[8] R. Schulz et al, "Network excitability in a model of chronic temporal lobe epilepsy critically depends on SK channel-mediated AHP currents," Neurobiol. Dis., vol. 45, no. 1, pp. 337-347, 2012.

[9] F. Frohlich, T. J. Sejnowski, and M. Bazhenov, "Network bistability mediates spontaneous transitions between normal and pathological brain states.," J. Neurosci., vol. 30, no. 32, pp. 10734-10743, Aug. 2010.

[10] G.-F. Tian et al, "An astrocytic basis of epilepsy.," Nat. Med., vol. 11, no. 9, pp. 973-81, 2005.

[11] L. Oyehaug et al, "Dependence of spontaneous neuronal firing and depolarisation block on astroglial membrane transport mechanisms," $J$. Comput. Neurosci., vol. 32, no. 1, pp. 147-165, 2012.

[12] H. Kager, W. J. Wadman, and G. G. Somjen, "Seizure-like afterdischarges simulated in a model neuron," J. Comput. Neurosci., vol. 22, no. 2, pp. 105-128, 2007.

[13] G. Ullah and S. J. Schiff, "Assimilating seizure dynamics," PLoS Comput. Biol., vol. 6, no. 5, pp. 1-12, 2010.

[14] G. P. Krishnan and M. Bazhenov, "Ionic dynamics mediate spontaneous termination of seizures and postictal depression state.," J Neurosci, vol. 31, no. 24, pp. 8870-8882, 2011.

[15] G. P. Krishnan et al, "Electrogenic properties of the $\mathrm{Na}^{+} / \mathrm{K}^{+}$ATPase control transitions between normal and pathological brain states.," $J$. Neurophysiol., vol. 113, no. 9, pp. 3356-74, 2015.

[16] J. R. Cressman et al, "The influence of sodium and potassium dynamics on excitability, seizures, and the stability of persistent states: I. Single neuron dynamics," J. Comput. Neurosci., vol. 26, no. 2, pp. 159-170, 2009.

[17] N. Hubel, R. D. Andrew, and G. Ullah, "Large extracellular space leads to neuronal susceptibility to ischemic injury in a $\mathrm{Na}^{+} / \mathrm{K}^{+}$pumpsdependent manner," J. Comput. Neurosci., vol. 40, no. 2, pp. 177-192, 2016.

[18] Y. Wei et al, "Oxygen and seizure dynamics: II. Computational modeling.," J. Neurophysiol., vol. 112, no. 2, pp. 213-23, 2014.

[19] M. Cotic et al, "Common time-frequency analysis of local field potential and pyramidal cell activity in seizure-like events of the rat hippocampus," J. Neural Eng., vol. 8, p. 046024, 2011.

[20] O. C. Zalay et al, "System characterization of neuronal excitability in the hippocampus and its relevance to observed dynamics of spontaneous seizure-like transitions.," J. Neural Eng., vol. 7, p. 036002, 2010.

[21] E. N. Brown, "'Theory of Point Processes for Neural Systems," Methods and Models in Neurophysics, pp. 691-727, 2005.

[22] A. C. Smith and E. N. Brown, "Estimating a State-Space Model from Point Process Observations," Neural Comput, vol. 15, no. 5, pp. 965-991, 2003.

[23] K. A. C. Ferguson and Sue Ann, "A two compartment model of a CA1 pyramidal neuron," Canadian Applied Mathematics Quarterly, vol. 17, pp. 293-302, 2009.

[24] K. M. L. Menne, T. Malina, and U. G. Hofmann, "Biologically realistic simulation of a part of hippocampal CA3: Generation of testdata for the evaluation of spike detection algorithms," Fifth GWAL, pp. 17-25, 2002.

[25] J.M. Bower, D. Beeman, The Book of GENESIS: Exploring Realistic Neural Models with the GEneral NEural SImulation System, Second Edition, Springer-Verlag, 1998.

[26] R. D. Traub et al, "A model of a CA3 hippocampal pyramidal neuron incorporating voltage-clamp data on intrinsic conductances.," J. Neurophysiol., vol. 66, no. 2, pp. 635-650, 1991.
[27] A. Sargsyan et al, "Simulation of sleep spindles and spike and wave discharges using a novel method for the calculation of field potentials in rats," J. Neurosci. Methods, vol. 164, no. 1, pp. 161-176, 2007.

[28] C. Giaume et al, "Astroglial networks: a step further in neuroglial and gliovascular interactions," Nat Rev Neurosci, vol. 11, no. 2, pp. 87-99, 2010.

[29] R. Nunez, P.L. and Srinivasan, Electric Fields of the Brain, 2nd ed. Oxford,UK: Oxford university press, 2005.

[30] M. Bikson et al, "Depolarization block of neurons during maintenance of electrographic seizures.," J. Neurophysiol., vol. 90, no. 4, pp. 2402-2408, 2003.

[31] D. Bianchi et al, "On the mechanisms underlying the depolarization block in the spiking dynamics of CA1 pyramidal neurons," J. Comput. Neurosci., vol. 33, no. 2, pp. 207-225, 2012.

[32] I. Osorio et al, Epilepsy: The Intersection of Neurosciences, Biology, Mathematics, Engineering, and Physics. 2016.

[33] E. E. Kang et al, "Markers of pathological excitability derived from principal dynamic modes of hippocampal neurons.," J. Neural Eng., vol. 9, no. 5, p. 056004, 2012.

[34] O. C. Zalay, "Cognitive Rhythm Generators for Modelling and Modulation of Neuronal Electrical Activity," University of Toronto, pp. 248-249, 2011.

[35] S. Colic et al, "Characterization of seizure-like events recorded in vivo in a mouse model of Rett syndrome," Neural Networks, vol. 46, pp. 109$115,2013$.

[36] S. Colic et al, "Characterization of HFOs in short and long duration discharges recorded from in-vivo MeCP2-deficient mice," IEEE Eng. Med. Biol. Soc. Annu. Conf., vol. 2014, pp. 4603-4606, 2014.

[37] A. B. L. Tort et al, "Measuring Phase-Amplitude Coupling Between Neuronal Oscillations of Different Frequencies," J. Neurophysiol., vol. 104, no. 2, pp. 1195-1210, 2010.

[38] N. B. Fountain et al, "Responses of Deep Entorhinal Cortex are Epileptiform in an Electrogenic Rat Model of Chronic Temporal Lobe Epilepsy," J Neurophysiol., vol. 80, no. 1, pp. 230-40, 1998

[39] B. D. Wissel et al, "Which patients with epilepsy are at risk for psychogenic nonepileptic seizures (PNES) - A multicenter case-control study," Epilepsy Behav., vol. 61, pp. 180-184, 2016.

[40] P. R. Bauer et al, "Dynamics of convulsive seizure termination and postictal generalized EEG suppression", Brain, vol. 140, pp. 655-668, 2017.

[41] P. Suffczynski et al, "Dynamics of Epileptic Phenomena Determined From Statistics of Ictal Transitions", IEEE Trans. Biomed. Eng., vol. 53, no. 3, pp. 524-532, Mar. 2006.

[42] L. Chunsheng et al, "Epileptogenic Source Imaging Using Cross Frequency Coupled Signals from Scalp EEG," IEEE Trans. Biomed. Eng., vol. 63, no. 12, pp. 2607-2618, Dec. 2016.

[43] G. J. Gutierrez, T. O'Leary, and E. Marder, "Multiple Mechanisms Switch an Electrically Coupled, Synaptically Inhibited Neuron between Competing Rhythmic Oscillators," Neuron, vol. 77, pp. 845-858, 2013.

[44] G. Buzsaki, A. Draguhn, "Neuronal oscillations in cortical networks," Science, vol. 304, pp.1926-1929, 2004.

[45] Z. J. Zhang et al, "Transition to Seizure: Ictal Discharge Is Preceded by Exhausted Presynaptic GABA Release in the Hippocampal CA3 Region," J. Neurosci., vol. 32, no. 7, pp. 2499-2512, 2012.

[46] D. W. Hochman, "The extracellular space and epileptic activity in the adult brain: Explaining the antiepileptic effects of furosemide and bumetanide," Epilepsia, vol. 53, no. SUPPL. 1, pp. 18-25, 2012.

[47] P. Bazzigaluppi et al, "Astrocytic gap junction blockade markedly increases extracellular potassium without causing seizures in the mouse neocortex," Neurology of Disease, vol. 101, pp. 1-7, 2017.

[48] V. Grigorovsky and B. L. Bardakjian, "Balance of synaptic and electrotonic connections controls the excitability of networks in biophysical model of epilepsy," IEEE Eng. Med. Biol. Soc. Annu. Conf., vol. 2015, pp. 4709-4712. 